\title{
Clear salt water above sediment-laden fresh water: Interfacial instabilities
}

\author{
B. Schulte, N. Konopliv, and E. Meiburg* \\ Department of Mechanical Engineering, University of California, Santa Barbara, Santa Barbara, \\ California 93106-5070, USA
}

(Received 16 February 2016; published 2 May 2016)

\begin{abstract}
The stability of an interface separating less dense, clear salt water above from more dense, sediment-laden fresh water below is explored via direct numerical simulations. We find that the destabilizing effects of double diffusion and particle settling amplify each other above the diffusive interface, whereas they tend to cancel each other below. For moderate settling velocities, plumes form both above and below the interface, whereas for large settling velocities plume formation below the interface is suppressed. We identify the dimensionless parameter that determines in which regime a given flow takes place, along with the critical value at which the transition between the regimes takes place.
\end{abstract}

DOI: 10.1103/PhysRevFluids.1.012301

\section{INTRODUCTION}

Recent investigations by Burns and Meiburg [1,2] and Yu et al. [3,4] address the instability of an interface separating a less dense layer of sediment-laden fresh water above from a more dense layer of clear salt water below. This configuration is introduced as a conceptual model for buoyant river plumes or hypopycnal turbidity currents propagating along the ocean's surface, respectively. The evolution of the interface is found to be governed by the competition of two linear instability modes. On the one hand, the unstable stratification of the more slowly diffusing particulate phase can trigger double-diffusive convection within the interfacial region. On the other hand, the downward settling of the particular phase prompts the formation of a maximum-density nose layer of sediment-laden salt water along the interface, which can trigger the growth of a Rayleigh-Taylor instability along its lower boundary. Direct numerical simulations show that the effective thicknesses $l_{s}$ and $l_{c}$ of the salinity and sediment concentration interfaces grow proportionally to the square root of time, as does the nose layer thickness $H$, so these processes can be modeled in terms of a turbulent diffusivity. For small values of $H / l_{s}<O(0.1)$ double-diffusive fingering is found to dominate, whereas for larger ratios $H / l_{s}>O(0.1)$ the dominant instability is of Rayleigh-Taylor type. This finding is consistent with the experimental observations by Parsons et al. [5].

It is well known that under exceptional discharge conditions some rivers can carry sufficiently high sediment loads to render them denser than the saline ocean water [6]. Under these conditions, the river plume will propagate along the seafloor (hyperpycnal flow) so that a layer of more dense, sediment-laden fresh water is situated below less dense, clear salt water [7] [see Fig. 1(a)]. The downward particle settling velocity is now directed away from the interface so that it favors the formation of a minimum-density nose region along the interface, with the potential for RayleighTaylor instability along its upper boundary, as sketched in Fig. 1(b). Furthermore, the differential diffusion of salt (fast) and sediment (slow) tends to create locally unstable density overhangs along both the upper and the lower boundaries of the interfacial region, which will give rise to gravitational instabilities. This regime of double-diffusive instability, in which the more slowly diffusing particular phase is stably stratified, is commonly referred to as the diffusive type [8-11] [see Fig. 1(c)]. Thus, the superposition of double-diffusive and settling effects amplifies the potential for Rayleigh-Taylor instability above the interface and reduces it below [see Fig. 1(d)]. The present investigation aims to explore the nonlinear competition among these instability modes via direct numerical simulations

*meiburg@engineering.ucsb.edu 


\section{B. SCHULTE, N. KONOPLIV, AND E. MEIBURG}
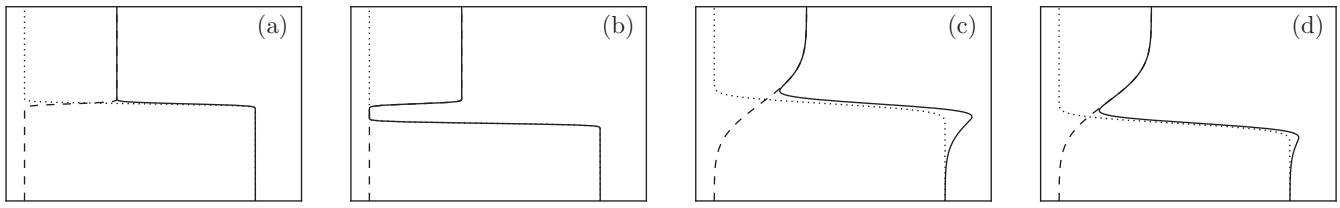

FIG. 1. Qualitative shape of salinity (dashed line), sediment (dotted line), and total (solid line) density distributions under various conditions: (a) initial distribution; (b) later time, as a result of particle settling only; (c) later time, as a result of different diffusivities only; and (d) later time, as a result of both settling and different diffusivities.

(DNSs), with a particular focus on the conditions under which the gravitational instability below the interface will be suppressed. While we will generally refer to the more rapidly diffusing scalar as salt, we note that similar considerations apply to the inflow of a denser, warm, sediment-laden river into a less dense, cold, fresh water lake.

\section{GOVERNING EQUATIONS AND INITIAL AND BOUNDARY CONDITIONS}

The governing equations are identical to the ones employed by Burns and Meiburg [2] and we refer the reader to this reference for the detailed derivation. The equations are made dimensionless using as salinity scale $S_{\max }$ the initial salinity in the upper layer and as particle concentration scale $C_{\max }$ the initial concentration in the lower layer. In addition, we employ viscous scales for length $d=\left(\frac{v^{2}}{g \gamma C_{\max }}\right)^{1 / 3}$, time $d^{2} / v$, and velocity $v / d$, reflecting a balance of inertial, viscous, and buoyancy forces. Here $\alpha$ and $\gamma$ are the expansion coefficients for salinity and sediment concentration, respectively. In dimensionless form, the conservation equations for mass, momentum, salinity $S$, and sediment concentration $C$ are

$$
\begin{aligned}
\nabla \cdot \boldsymbol{u} & =0, \\
\frac{\partial \boldsymbol{u}}{\partial t}+\boldsymbol{u} \cdot \nabla \boldsymbol{u} & =\nabla^{2} \boldsymbol{u}-\nabla P-\left(R_{S} C+S\right) \hat{\boldsymbol{y}}, \\
\frac{\partial S}{\partial t}+\boldsymbol{u} \cdot \nabla S & =\frac{1}{\mathrm{Sc}} \nabla^{2} S, \\
\frac{\partial C}{\partial t}+V_{p} \frac{\partial C}{\partial y}+\boldsymbol{u} \cdot \nabla C & =\frac{1}{\tau \mathrm{Sc}} \nabla^{2} C,
\end{aligned}
$$

with the governing dimensionless parameters

$$
V_{p}=\frac{v_{s}}{\left(\nu g \alpha S_{\max }\right)^{1 / 3}}, \quad \mathrm{Sc}=\frac{\nu}{\kappa_{s}}, \quad \tau=\frac{\kappa_{s}}{\kappa_{c}}, \quad R_{s}=\frac{\gamma C_{\max }}{\alpha S_{\max }} .
$$

Here the dimensionless settling velocity $V_{p}$ represents the ratio of the Stokes settling velocity $v_{s}$ and the characteristic viscous buoyancy velocity; Sc denotes the Schmidt number, which is based on the diffusivity of salinity $\kappa_{s} ; \kappa_{c}$ refers to the sediment diffusivity; and the diffusivity ratio $\tau>1$. The upper layer being less dense than the lower layer, the stability ratio $R_{s}$, which represents the ratio of the density contributions of sediment and salt, is always larger than unity in the present investigation.

Based on a typical seawater salinity of $3.5 \%$, a typical velocity scale in this problem is about $7 \mathrm{~mm} / \mathrm{s}$ or less. In this study, we analyze a simulation with $V_{p}=0.04$, which corresponds to a grain size of $9.0 \mu \mathrm{m}$, a fine silt, assuming a particle specific gravity of 2.6 along with the Stokes law for the settling velocity. Depending on the sediment content of the fresh water, the stability ratio $R_{S}$ can take on a range of values in nature. Here we employ $R_{s}=2.5$ so that the sediment loading is 2.5 times larger than the salinity loading. While $\mathrm{Sc}=700$ for salt water, this value is too large to conduct DNSs and we use a value of 1 instead. Estimates for the physical diffusivity of the particles 
as a function of their size are available in the literature [12]. Here we use $\tau=25$ and note that further increases in $\tau$ made little difference in the final results.

The present investigation employs two-dimensional simulations of the above equations. We note that for the case of fresh water with particles above salt water, Burns and Meiburg [2] had observed similar dynamics in two- and three-dimensional simulations, so we can expect two-dimensional simulations to provide meaningful insight for the present case. Our simulations use periodic boundary conditions in the horizontal direction and slip walls with no flux at the top and bottom boundaries. The simulations are always terminated before any plumes reach these boundaries. The flow field is initialized from rest, with randomly perturbed, slightly smoothed step profiles for sediment and salinity.

\section{DIRECT NUMERICAL SIMULATIONS}

In order to demonstrate the effects of settling and double diffusion in isolation, Figs. 2 and 3 present the salinity and sediment concentration fields for four representative simulations with $R_{s}=2.5$, at three different times. While the full $x$ axis is displayed, the domain is truncated in the $y$ direction, so we can focus on the most active region near the interface. The left column shows the case of particle settling in the absence of double diffusion, for $V_{p}=0.04$, Sc $=25$, and $\tau=1$. Rising plumes can
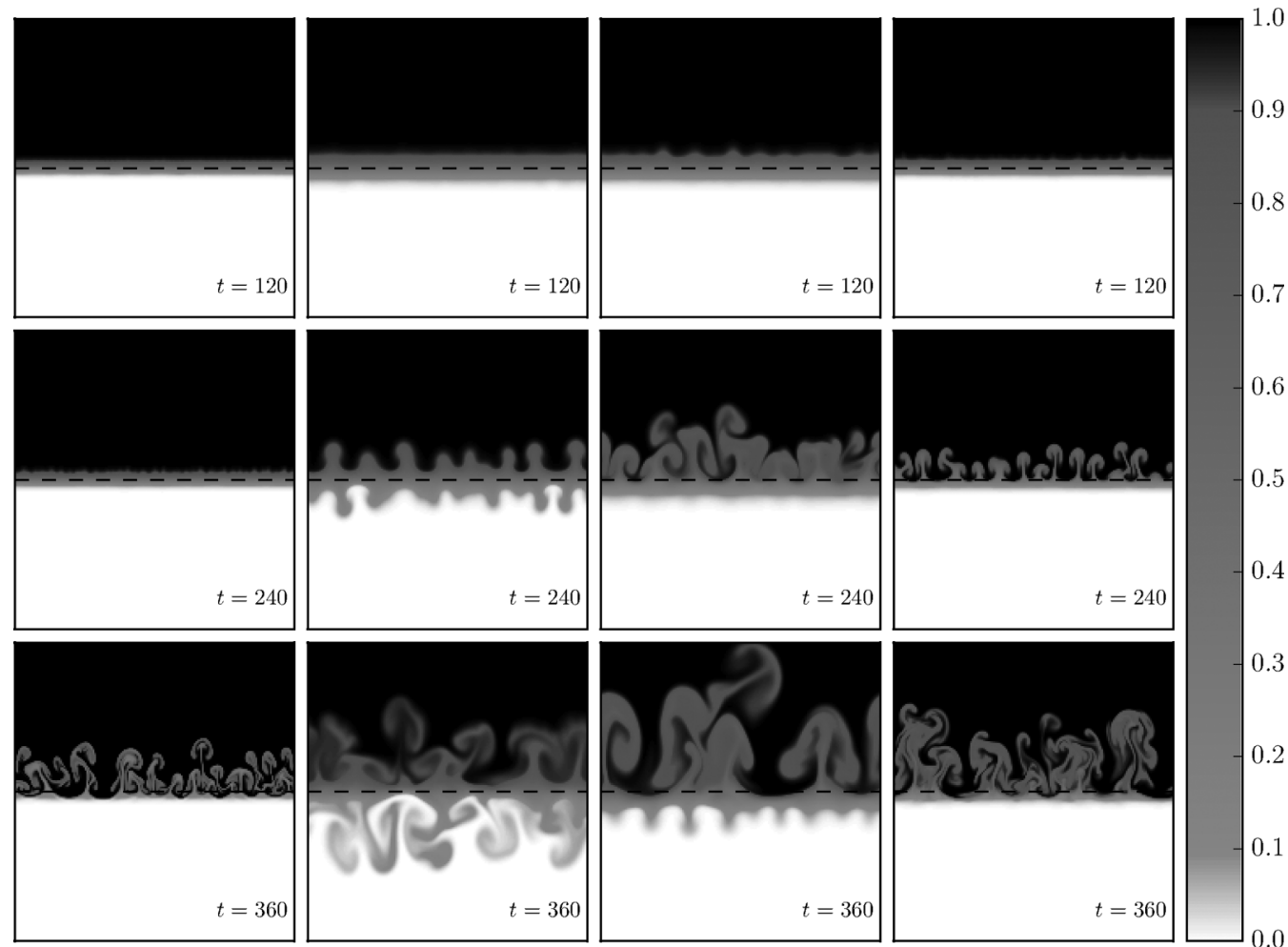

FIG. 2. Snapshots of salinity concentration fields for four simulations with $R_{s}=2.5$. Shown in the first column from the left is the effect of settling only, for $V_{p}=0.04, \mathrm{Sc}=25$, and $\tau=1$; in the second column, the effect of double diffusion only, for $V_{p}=0, \mathrm{Sc}=1$, and $\tau=25$; the third column, the combined effects of settling and double diffusion, for $V_{p}=0.04, \mathrm{Sc}=1$, and $\tau=25$; and the rightmost column, the effect of settling is strong enough to eliminate plumes in the bottom half, for $V_{p}=0.04, \mathrm{Sc}=7$, and $\tau=25$. The domain shown extends from 0 to 700 in $x$ and -300 to 300 in $y$, with the initial interface at $y=0$ marked with a dashed line. 

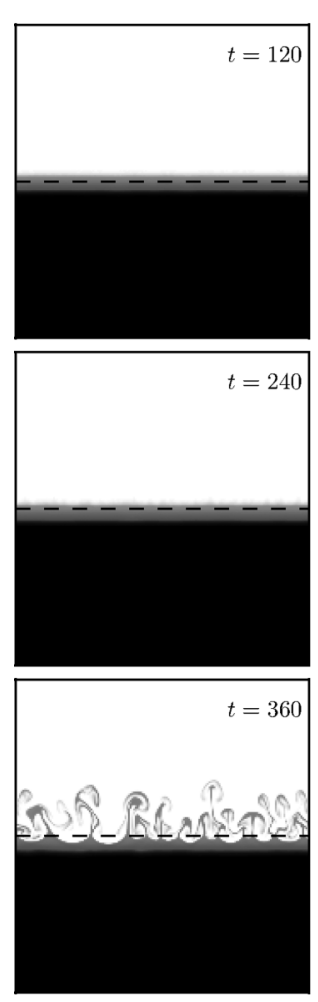
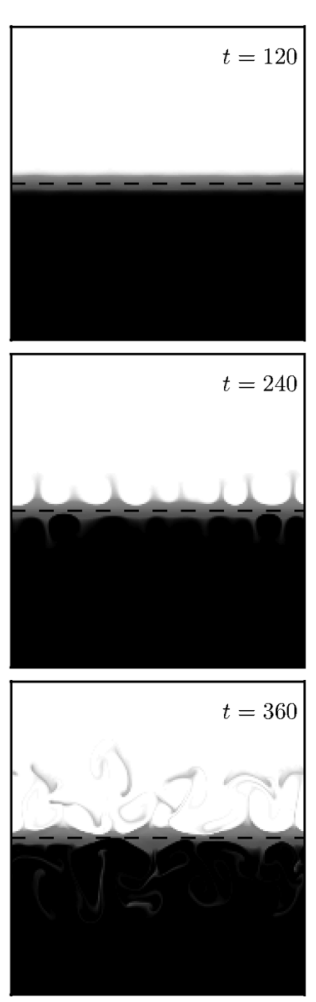
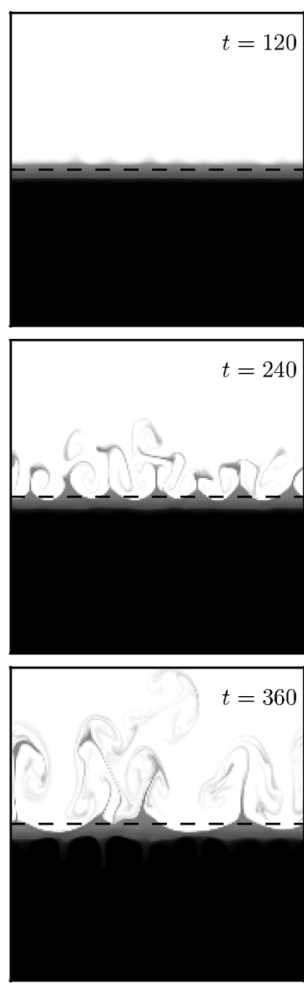

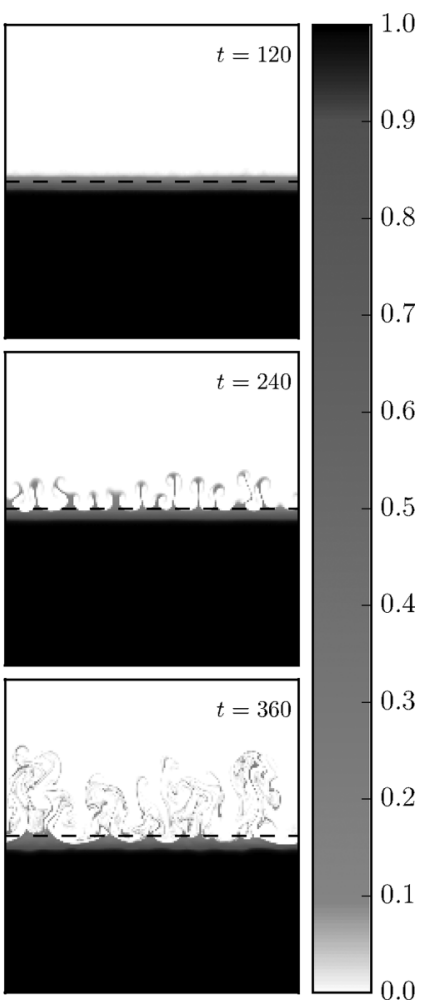

FIG. 3. Snapshots of sediment concentration fields for three simulations with $R_{s}=2.5$. Shown in the first column from the left is the effect of settling only, for $V_{p}=0.04, \mathrm{Sc}=25$, and $\tau=1$; in the second column, the effect of double diffusion only, for $V_{p}=0, \mathrm{Sc}=1$, and $\tau=25$; the third column, the combined effects of settling and double diffusion, for $V_{p}=0.04, \mathrm{Sc}=1$, and $\tau=25$; and the rightmost column, the effect of settling is strong enough to eliminate plumes in the bottom half, for $V_{p}=0.04, \mathrm{Sc}=7$, and $\tau=25$. The domain extends from 0 to 700 in $x$ and -300 to 300 in $y$, with the initial interface at $y=0$ marked with a dashed line.

be identified in both the salinity and sediment concentration fields, as a result of a Rayleigh-Taylor instability that develops along the upper boundary of the interfacial region, in accord with the sketch in Fig. 1(b). By contrast, the second column shows the case of double-diffusive instability in the absence of particle settling, for $V_{p}=0, \mathrm{Sc}=1$, and $\tau=25$. Consistent with the sketch in Fig. 1(c), the flow exhibits a diffusively dominated central layer from which positively and negatively buoyant plumes of roughly equal size rise and descend into the upper and lower regions, as a result of the local density overhangs above and below the central layer, respectively. The third column shows the combined effects of double diffusion and sedimentation, for $V_{p}=0.04, \mathrm{Sc}=1$, and $\tau=25$. As expected from Fig. 1(d), we recognize a combination of strong rising and weaker descending plumes, which indicates that sedimentation destroys the approximate symmetry of the pure double-diffusive instability. Finally, the right column shows a simulation for $V_{p}=0.04, \mathrm{Sc}=7$, and $\tau=25$, for which the settling is sufficiently strong to suppress the formation of plumes below the interfacial region. For those flows with $\tau=25$, the sediment concentration gradients are much more pronounced than the salinity ones, due to the smaller diffusivity of the sediment. Taken together, Figs. 2 and 3 indicate that along the upper boundary of the central, diffusively dominated region the effects of double-diffusion and sedimentation amplify each other, whereas along its lower boundary they tend to cancel each other, possibly even to the point where instabilities in the lower fluid layer are completely suppressed, which is consistent with the sketches of Fig. 1. 

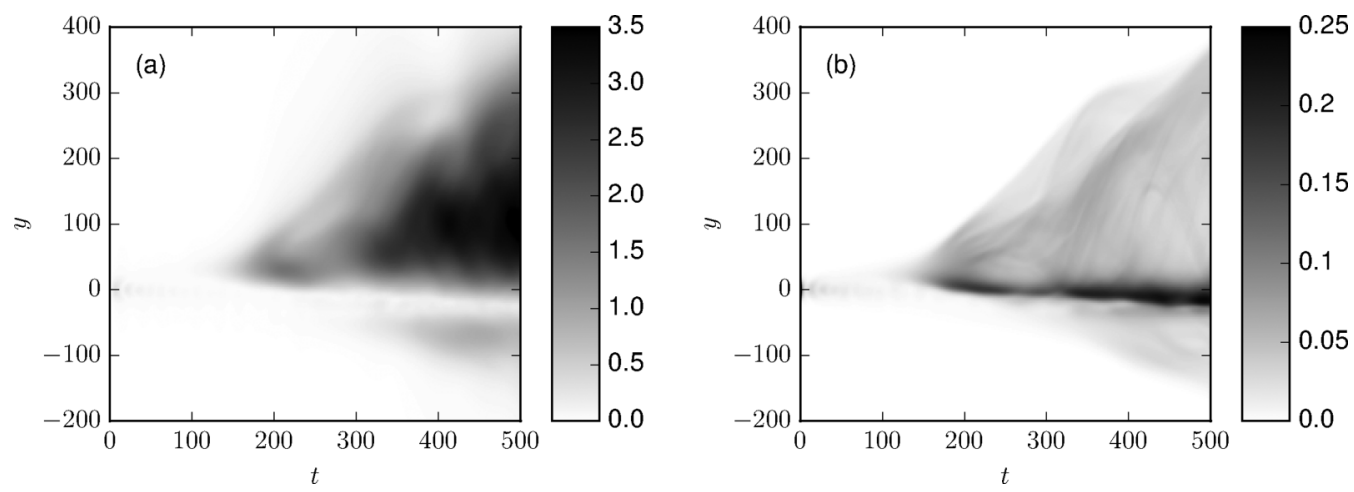

FIG. 4. (a) Horizontal rms fluctuations of the salinity concentration field as a function of the vertical coordinate and time. (b) Horizontal rms fluctuations of the vertical velocity as a function of the vertical coordinate and time. The parameters for this simulation were $\mathrm{Sc}=1, R_{s}=2.5, \tau=25$, and $V_{p}=0.04$.

In the following, our analysis will focus on the simulation shown in the third columns of Figs. 2 and 3, where both sedimentation and double diffusion are influential. Figure 4(a) displays the horizontal rms fluctuations of the salinity field, as a function of the vertical location and time. It confirms that the rising plumes above the interface are stronger and spread more rapidly than their descending counterparts below the interface. The analogous plot for the sediment concentration field (not shown) displays corresponding features. The horizontal rms fluctuations of the vertical velocity field for the present case, shown in Fig. 4(b), exhibit characteristics that are quite different from the configuration analyzed by Burns and Meiburg [2]. These fluctuations can be seen to be very small in the slowly descending, central interfacial region, confirming that this region is dominated by diffusion rather than convection. This suggests that the flux of sediment across the central region will be a result of diffusion and settling only, while the transport of salinity will be purely diffusive, and the convective components are negligible.

These differences between the two configurations are confirmed by the temporal evolution of the sediment and salinity interface thicknesses $l_{c}(t)$ and $l_{s}(t)$ as well as the corresponding interface locations $y_{c}(t)$ and $y_{s}(t)$ (see Fig. 5). These quantities are obtained by fitting the horizontally averaged concentration profiles $\bar{S}(y, t)$ and $\bar{C}(y, t)$ to error functions (see Ref. [2]). Equation (6) shows the
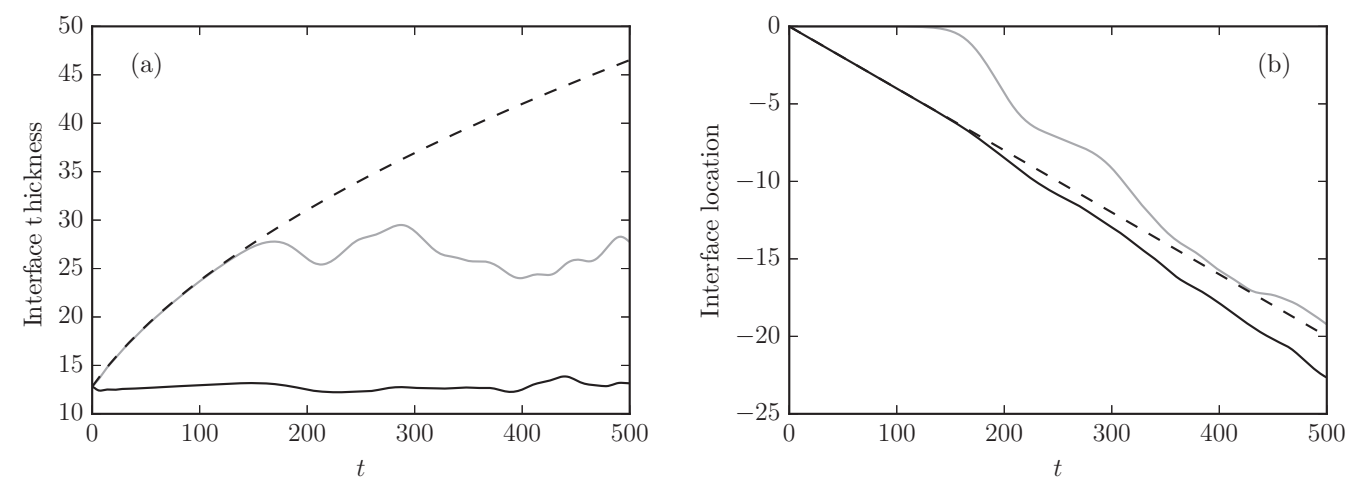

FIG. 5. (a) Interface thicknesses for sediment (black) and salinity (gray) as functions of time. The dashed line represents the initial diffusive growth of the salinity interface thickness according to $\sqrt{4 t / \mathrm{Sc}}$. (b) Interface locations for sediment (black) and salinity (gray) as functions of time. The dashed line represents the Stokes settling velocity. The parameters for this simulation were $\mathrm{Sc}=1, R_{s}=2.5, \tau=25$, and $V_{p}=0.04$. 


\section{B. SCHULTE, N. KONOPLIV, AND E. MEIBURG}

form of the fitted function for salinity, with an identical expression holding for particle concentration

$$
\bar{S}(y, t)=\frac{1}{2} \operatorname{erf}\left(\frac{y-y_{s}(t)}{l_{s}(t)}\right)
$$

The inflection point of the error function profile then indicates the interface location, while the error function width is taken as the effective interface thickness. Burns and Meiburg [2] found that for sediment-laden fresh water above salt water both interface thicknesses grew proportionally to $t^{1 / 2}$ for all times, so effective turbulent diffusivities could be defined. Their simulation results showed that, in spite of its much larger molecular diffusivity, the turbulent diffusivity of salt was smaller than that of sediment. Figure 5(a) demonstrates that, for the present configuration of clear salt water above sediment-laden fresh water, the salinity interface thickness grows diffusively only during the early stages of the flow. Once the convective instability sets in, both interface thicknesses fluctuate around values that remain constant in time. This reflects a competition between diffusion, which tends to thicken the interface, and convection, which continuously sharpens it, analogous to a thermal boundary layer [10].

For sediment-laden fresh water above salt water, Burns and Meiburg [2] found that the sediment interface location, after initially descending with the particle settling velocity, propagates downward at a reduced rate, while the salinity interface location moves upward. As a result, the distance between the salinity and particle interfaces, i.e., the nose thickness $H$, was seen to grow proportionally to $t^{1 / 2}$. A qualitatively different picture emerges for the configuration of clear salt water above sediment-laden fresh water [see Fig. 5(b)]. Here the sediment interface propagates downward roughly with the settling speed for all times. We note that the downward velocity of the sediment interface is not to be confused with the effective particle settling velocity, which varies depending on whether or not downward moving plumes are present. The salinity interface location, after initially remaining stationary, subsequently tracks the sediment interface downward, so the distance between the two remains approximately constant with time. This quasisteady nose thickness can be understood as the result of a balance between two competing effects: The continued settling of particles moves the sediment interface downward. This reduces the minimum density at the upper boundary of the interfacial region, which in turn increases the strength of the salinity convection above the interfacial region, thereby bringing the salinity interface downward. Once the salinity interface approaches the sediment interface, the density difference driving the convection above the interfacial region is reduced, the downward motion of the salinity interface slows, and the downward propagating sediment interface increases the offset again.
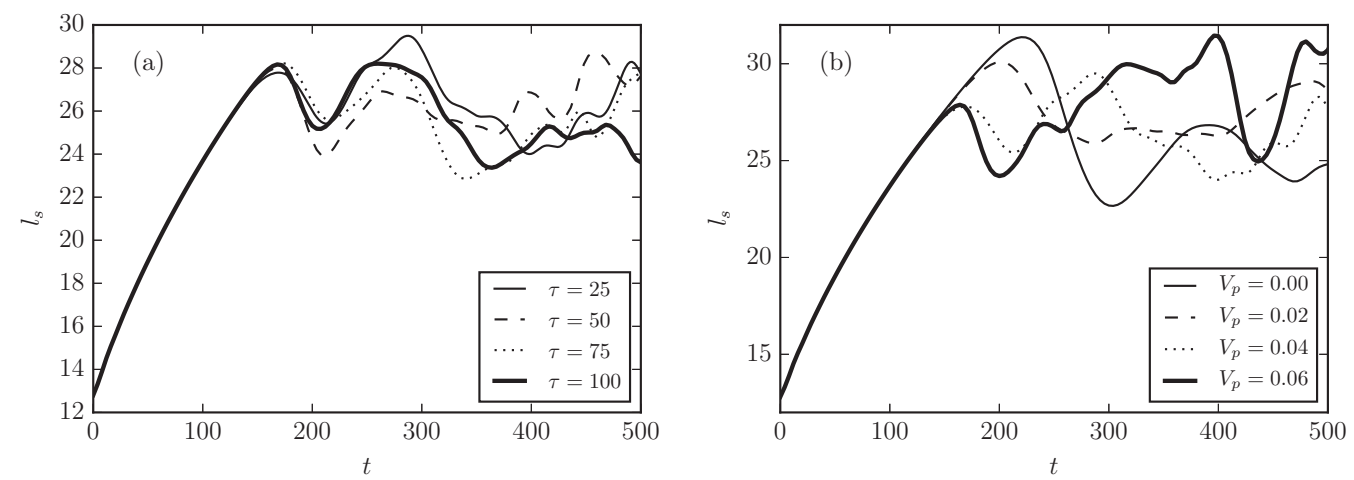

FIG. 6. (a) Development of $l_{s}$ over time for different values of $\tau$. (b) Development of $l_{s}$ over time for different settling velocities. The parameters are (a) $\mathrm{Sc}=1, R_{s}=2.5$, and $\tau=25,50,100,200$ and (b) $\mathrm{Sc}=1$, $R_{s}=2.5, \tau=25$, and $V_{p}=0,0.02,0.04,0.06$. 
CLEAR SALT WATER ABOVE SEDIMENT-LADEN FRESH ...

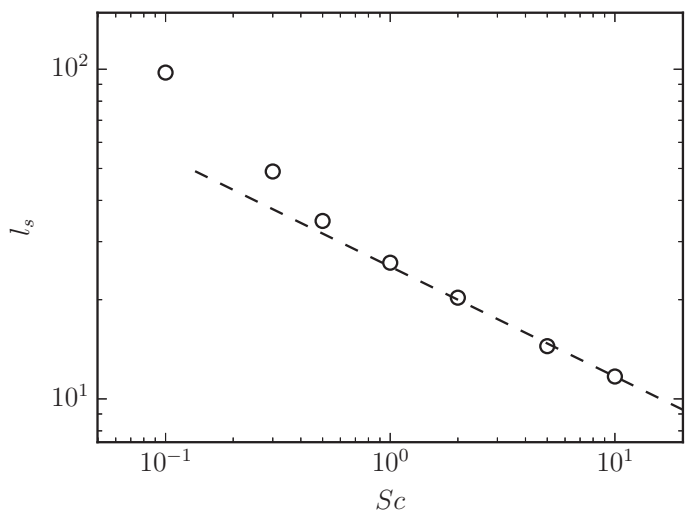

FIG. 7. Quasisteady salinity interface thickness $l_{s}$ for different values of the Schmidt number. The parameters are $\mathrm{Sc}=10,5,2,1,0.5,0.3,0.1, R_{s}=2.5, \tau=25$, and $V_{p}=0.04$. The dashed line has a slope of $-1 / 3$.

In the following, we will formulate scaling relations that allow us to distinguish the two main flow regimes, viz., the regime characterized by plumes both above and below the interfacial region and the one that exhibits plumes only above the interfacial region. The generation of plumes below the interface is linked to the emergence of a local density maximum in this region. In other words, descending plumes will form if the downward diffusive flux of salinity $F_{s} \propto 1 / \mathrm{Scl} l_{s}$ across the interfacial region exceeds the downward sediment flux as a result of settling $F_{c} \propto V_{p}$.

While Sc and $V_{p}$ are known input parameters, the quasisteady salinity interface thickness $l_{s}$ is not, so we have to obtain it from the numerical simulations. Towards this end, Fig. 6 shows a number of simulation results for $l_{s}(t)$ as a function of the diffusivity ratio $\tau$ and the settling velocity $V_{p}$. We find that, once convection sets in, $l_{s}$ remains approximately constant in time and depends only weakly on $\tau$ and $V_{p}$ for the regimes explored. On the other hand, $l_{s}$ depends strongly on the Schmidt number. A log-log plot of the quasisteady $l_{s}$ as a function of Sc shows that $l_{s} \propto \mathrm{Sc}^{-1 / 3}$ for large Sc (see Fig. 7). A dimensional scaling argument in support of this relationship can be constructed along

TABLE I. Values of Sc and $V_{p}$ from the simulations in Fig. 8.

\begin{tabular}{lcr}
\hline \hline Simulation No. & $V_{p}$ & $\mathrm{Sc}$ \\
\hline 1 & 0.02 & 1.0 \\
2 & 0.04 & 1.0 \\
3 & 0.06 & 1.0 \\
4 & 0.08 & 1.0 \\
5 & 0.04 & 7.0 \\
6 & 0.04 & 5.0 \\
7 & 0.04 & 2.0 \\
8 & 0.04 & 0.5 \\
9 & 0.04 & 0.1 \\
10 & 0.03 & 3.0 \\
11 & 0.05 & 3.0 \\
12 & 0.02 & 4.0 \\
13 & 0.01 & 6.0 \\
14 & 0.04 & 10.0 \\
15 & 0.04 & 9.0 \\
\hline \hline
\end{tabular}




\section{B. SCHULTE, N. KONOPLIV, AND E. MEIBURG}

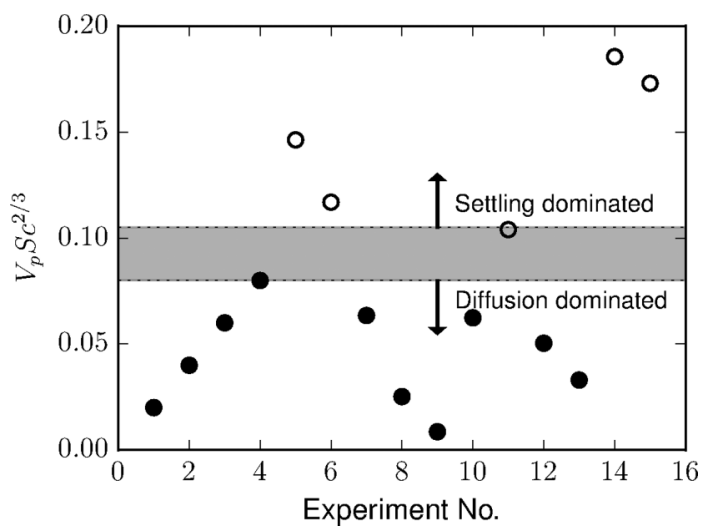

FIG. 8. Value of the ratio of the sediment to salinity flux $V_{p} \mathrm{Scl}_{s}$ for 15 numerical simulations with $R_{s}=2.5$, $\tau=25$, and the Sc and $V_{p}$ values listed in Table I. Open markers indicate that plumes do not form below the interface, while closed markers reflect the existence of plumes in this region.

the following lines: The plumes emanating from the interface at discrete locations generate shear along the interface, thereby setting up a viscous boundary layer along the horizontal interface. Since we employed viscous scaling to derive the original set of governing equations, we have $u \propto \frac{v}{\delta}$ within this boundary layer. For large $\mathrm{Sc}$ the salinity boundary layer of thickness $l_{s}$ will be much thinner than the viscous boundary layer of thickness $\delta$. Hence we have a characteristic velocity of the salinity $u_{s}=\frac{u l_{s}}{\delta}=\frac{\nu l_{s}}{\delta^{2}}$. Balancing the dominant convective and diffusive terms in the salinity equation $u_{s} \frac{\partial S}{\partial x}$ and $k_{s} \frac{\partial^{2} S}{\partial y^{2}}$, respectively, then yields $\frac{l_{s}}{\delta} \propto\left(\frac{k_{s}}{v}\right)^{1 / 3}=S c^{-1 / 3}$, in agreement with the simulation data in Fig. 7. This scaling is only valid for moderate and large Sc, since when $\mathrm{Sc}$ is small salinity will tend to diffuse further than the viscous boundary layer and then the characteristic velocity of the salinity will no longer be $u_{s} \propto \frac{u l_{s}}{\delta}$.

In order to explore the existence of a critical value of $V_{p} \mathrm{Scl} l_{s}$ beyond which the formation of plumes below the interface would be suppressed, we carried out simulations for $R_{s}=2.5, \tau=25$, and the Sc and $V_{p}$ values listed in Table I. Figure 8 indicates whether or not a specific simulation exhibits negatively buoyant plumes. The graph confirms that for $V_{p} \mathrm{Scl} l_{s} \leqslant 2.5$ descending plumes form, whereas flows with $V_{p} \mathrm{Scl}_{s} \geqslant 2.5$ exhibit rising plumes only.

It will be of interest to explore how the above scaling law will need to be modified in the presence of shear across the interface.

\section{ACKNOWLEDGMENTS}

The authors thank Professor Pascale Garaud and Professor Rama Govindarajan for helpful discussions. E.M. acknowledges support through NSF Grants No. CBET-1335148 and No. CBET-1438052.

[1] P. Burns and E. Meiburg, Sediment-laden fresh water above salt water: Linear stability analysis, J. Fluid Mech. 691, 279 (2012).

[2] P. Burns and E. Meiburg, Sediment-laden fresh water above salt water: Nonlinear simulations, J. Fluid Mech. 762, 156 (2015).

[3] X. Yu, T.-J. Hsu, and S. Balachandar, Convective instability in sedimentation: Linear stability analysis, J. Geophys. Res.-Oceans 118, 256 (2013). 


\section{CLEAR SALT WATER ABOVE SEDIMENT-LADEN FRESH ...}

[4] X. Yu, T.-J. Hsu, and S. Balachandar, Convective instability in sedimentation: 3-D numerical study, J. Geophys. Res.-Oceans 119, 8141 (2014).

[5] J. D. Parsons, J. W. M. Bush, and J. P. M. Syvitski, Hyperpycnal plume formation from riverine outflows with small sediment concentrations, Sedimentology 48, 465 (2001).

[6] T. Mulder and J. P. M. Syvitski, Turbidity currents generated at river mouths during exceptional discharges to the world oceans, J. Geol. 103, 285 (1995).

[7] R. S. J. Sparks, R. T. Bonnecaze, H. E. Huppert, J. R. Lister, M. A. Hallworth, H. Mader, and J. Phillips, Sediment-laden gravity currents with reversing buoyancy, Earth Planet. Sci. Lett. 114, 243 (1993).

[8] M. E. Stern, The salt-fountain and thermohaline convection, Tellus 12, 172 (1960).

[9] J. S. Turner, Double-diffusive phenomena, Annu. Rev. Fluid Mech. 6, 37 (1974).

[10] H. E. Huppert and J. S. Turner, Double-diffusive convection, J. Fluid Mech. 106, 299 (1981).

[11] T. Radko, Double-Diffusive Convection (Cambridge University Press, Cambridge, 2013).

[12] P. N. Segre, F. Liu, P. Umbanhowar, and D. A. Weitz, An effective gravitational temperature for sedimentation, Nature (London) 409, 594 (2001). 